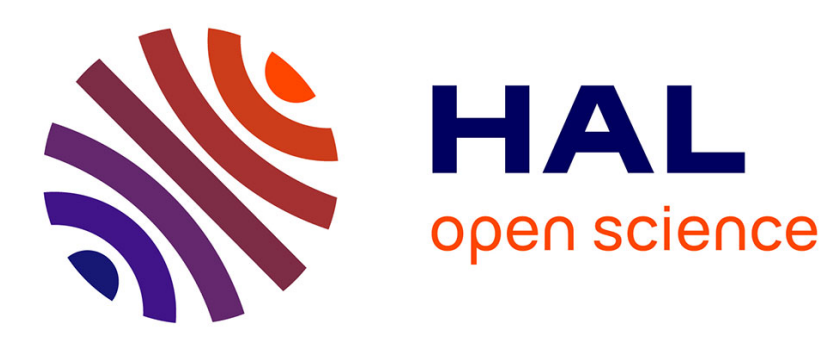

\title{
Frequency Reconfigurable Dual Narrow Band Antenna matched on the Low Band LTE FDD Physical Layer
}

\author{
Serge Bories, François Sarrazin, Alexandre Giry
}

\section{To cite this version:}

Serge Bories, François Sarrazin, Alexandre Giry. Frequency Reconfigurable Dual Narrow Band Antenna matched on the Low Band LTE FDD Physical Layer. 12th European Conference on Antennas and Propagation (EuCAP 2018), Apr 2018, London, United Kingdom. hal-01979434

\section{HAL Id: hal-01979434 \\ https://hal.science/hal-01979434}

Submitted on 12 Jan 2019

HAL is a multi-disciplinary open access archive for the deposit and dissemination of scientific research documents, whether they are published or not. The documents may come from teaching and research institutions in France or abroad, or from public or private research centers.
L'archive ouverte pluridisciplinaire $\mathbf{H A L}$, est destinée au dépôt et à la diffusion de documents scientifiques de niveau recherche, publiés ou non, émanant des établissements d'enseignement et de recherche français ou étrangers, des laboratoires publics ou privés. 


\title{
Frequency Reconfigurable Dual Narrow Band Antenna matched on the Low Band LTE FDD Physical Layer
}

\author{
S. Bories, F. Sarrazin, A. Giry \\ CEA LETI, UGA, Grenoble, France, serge.bories@ cea.fr
}

\begin{abstract}
The upcoming widening of the LTE frequency Low Band (LB) towards $600 \mathrm{MHz}$ imposes a strong miniaturization effort to the smartphone antenna which can no more maintain its radiation efficiency performance over the full 600-960 MHz range. To overcome this issue, the design strategy consists in matching the antenna only where it is instantaneously used. Thus, based on the FDD spectrum usage, the proposed antenna presents two narrow bands that can be reconfigured independently anywhere in the whole LTE LB. The proposed design takes into account current limitations of the tunable capacitor (low $Q$ factor). The measured total efficiency increases with frequency from $11 \%$ at $617 \mathrm{MHz}$ to $60 \%$ at $960 \mathrm{MHz}$.
\end{abstract}

Index Terms-reconfigurable antenna, dual narrow band, antenna miniatirisation.

\section{INTRODUCTION}

The properly named LTE (Long Term Evolution) standard supports ever increasing number of E-UTRA frequency bands. In the sub-GHz bands, this is particularly relevant for network providers to improve the coverage of mobile or IoT applications due to better EM propagation properties. For instance, from mid-2017, the E-UTRA band 71 [1] ( $2 \times 35 \mathrm{MHz}$ bandwidth starting from $617 \mathrm{MHz}$ ) operates in the USA. However, if the wavelength is increasing with coming bands, the terminal form-factor remains constant or even tends to shrink (cellular watch, NB-IoT sensor). These two challenges (lower band and smaller devices) can no more be faced with mere wideband passive antennas without deteriorating their performances (impedance matching and radiation efficiency).

In this context, a strategy to bypass the bandwidthminiaturization-efficiency trade-off is to give up the full 617$960 \mathrm{MHz}$ bandwidth (43\% fractional bandwidth) to focus only on single requested E-UTRA band. Indeed, this strategy is possible since so far the different Low Bands (LB) are not simultaneously used even during vertical handover or LTE inter-frequency handover. The intra LB carrier aggregation mode is not envisaged for the moment. However, it can be noticed that the proposed concept is compatible with inter band (LB/ High Band (HB) carrier aggregation since the HB is planned to be supported by a dedicated HB access (not shown in this paper).

Frequency agile antenna is not a new concept. Two different techniques are distinguished: the tunable impedance matching network (TMN) [2] and the Aperture Tuning [3] The former aims to match the antenna input impedance which has a high Q-factor in the case of electrically small antenna (ESA). Its real and imaginary parts are rapidly changing, with usually a strong capacitive behavior. For these reasons, the tuning range is limited. Conversely, the aperture tuning aims to shift the antenna resonance frequency itself, by implementing a reconfigurable lumped component or even substrate onto the radiating structure of the antenna. Thus, the frequency-agile antenna performance is mainly governed by the tunable component: its Q-factor and its position on the antenna structure. The design of a tunable digital capacitors array (MEMS, Integrated Circuit on SOI or SOS technologies, is also a trade-off between tuning range- Cmin-maximum $\mathrm{RF}$ power and its Equivalent Series Resistance (ESR) [4].

Recently, the challenge of tunable ESA design for LTE LB has been met with three categories of works: at the antenna level only, both at the TC and antenna level with a co-design approach, and at last thanks to system and RF Front-End Module (FEM) architecture considerations.

The first category merely deals with typical antenna miniaturization techniques and optimizes the position of a COTS TC: e.g. a DTC [5] or a varactor [6]. Interesting tuning range is reached at the cost of the instant bandwidth to cover both Up-Link (UL) and Down-Link (DL) in the lowest bands with a single resonance.

For extreme miniaturization (planar notch antenna in $\lambda / 26$ at $635 \mathrm{MHz}$ with $600-960 \mathrm{MHz}$ tuning range), the second category aims to distribute the constraints between ESA and a DTC in a co-design [4] [7]. It is shown that the tunable ESA radiation efficiency is equally affected by the finite conductivity of the antenna and the TC RF losses.

The last category leverages the LTE Frequency Division Duplex (FDD) spectrum access by designing two smaller and separated narrower bandwidth antennas: one for the UL and the second for the DL [8]. This spatial separation has a positive impact on the FEM (avoiding typical $2.5 \mathrm{~dB}$ duplexer IL). However, two areas are required on the cellular board side which may still raise some integration issues.

This paper synthesizes the main ideas of the three previous categories. The novelty of concept of the tunable dual narrow band antenna design is presented in chapter II. The TC ESR 
impact is demonstrated by simulation in chapter III. Then, the prototype is characterized in chapter IV.

\section{CONCEPT \& ANTENNA DESCRIPTION}

\section{A. Concept}

The Dual Tunable Bands Antenna (DTBA) implements the concept of aperture tuning twice. It is reminded that by designing the antenna over a narrower instant bandwidth, we can expect to maintain its radiation efficiency. To match the LTE FDD scheme, the innovation consists here by matching two independent resonances of the antenna over 2 x $10 \mathrm{MHz}$ : the first UL band, the second for the DL of the allocated frequency of a given network provider (Fig. 1). These two independent bands can be tuned over the whole LB. The duplex spacing can also be set accordingly to the selected EUTRA band.

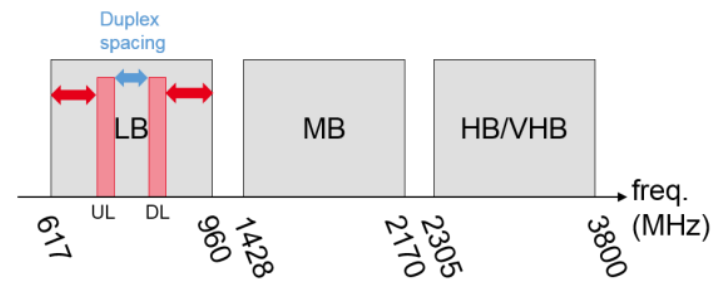

Fig. 1. Dual Tunable Bands Antenna covering two $10 \mathrm{MHz}$ instant bandwidth over the whole LTE LB

\section{B. Antenna description}

This design is inspired from the antenna in [5] where a meander monopole is coupled with a tunable parasitic element with a total volume of $40 \times 10 \times 6 \mathrm{~mm}^{3}$. In the same volume, we proposed to incorporate a second parasitic element to avoid the single resonance compromise in bandwidth. The two obtained narrow bands can be tuned regarding the RAT. The duplex spacing can be tuned between 30 and more than 60 MHz. The developed antenna is actually a folded monopole with less meander than in the original work. It is fed through a $50 \Omega \mathrm{CPW}$ line. This monopole is tightly coupled with two parasitic shorted loops whose electrical length can be tuned with a Switched Capacitor Array (SCA). For compactness reason, the structure is not symmetrical regarding the central monopole. As a consequence, the coupling is not the same for both parasitic elements.

The space dedicated to the antenna is extremely compact for the covered band (Fig.2). The radiating element is $40 \times 10$ x $6 \mathrm{~mm}^{3}$ (width, length, thick) over a PCB ground plane of $110 \times 80 \times 1.6 \mathrm{~mm}^{3}$. Two SCA with 5 bits states are controlled through one SPI bus with a dedicated Arduino shield and a STM32 nucleo (dual independent control). For the state 0, Cmin is $0,85 \mathrm{pF}$ and for the state $31, \mathrm{Cmax}$ is $2,4 \mathrm{pF}$. The mean ESR over the different states is $2.5 \Omega$.

Compared to the plane structures in SotA, the proposed antenna takes benefit of the available volume, which is useful to improve the ESA total efficiency. Secondly, the position of the tunable element on the coupled parasite has been optimized to reduce the current flowing through the SCA. This aims to lower the impact on efficiency of the SCA ESR as explained in next section.

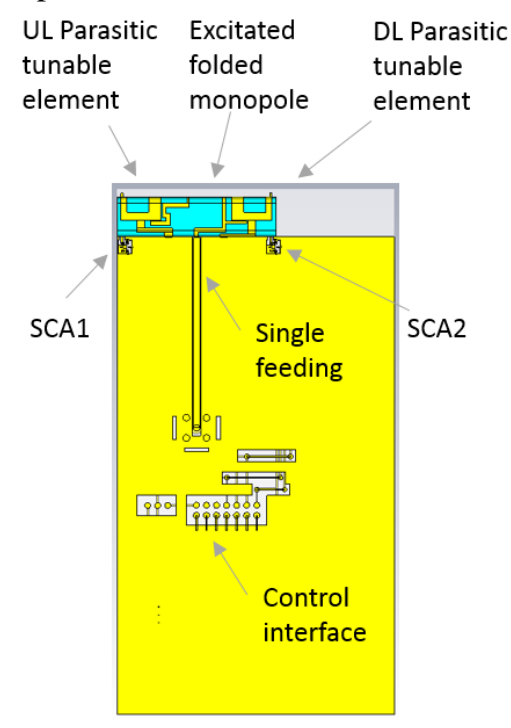

(a)

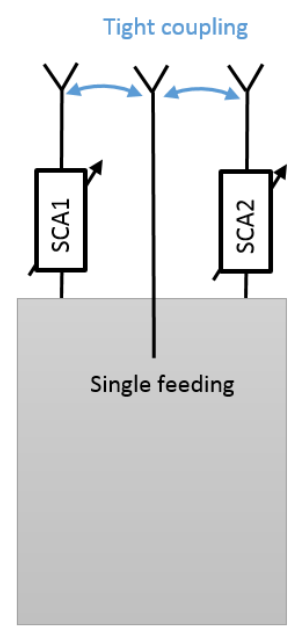

(b)
Fig. 2. DTBA structure with realisation details (a) and its RF principle scheme (b)

\section{IMPACT OF RECONFIGURABLE COMPONENT ESR}

Previous studies have highlighted the trade-off between the radiation efficiency and antenna miniaturization through the coupling between monopoles. If the former is too low, the miniaturization is lost. Inversely if the coupling is too high (closer inter element spacing), the current flowing into the SCA is very high; thus the total radiation efficiency is really impacted by the ESR of the tuned elements. This conclusion emphasizes the mutual roles of the antenna design and the SCA design (via its ESR).

The CST MWS simulated radiation performance of the DTBA is plotted in Figure 3. Impedance mismatch losses are not taken into account to simplify the analysis. The IEEE radiation efficiencies are compared for three settings of UL/DL bands: 900-960 MHz, 700-760 MHz, and 600$660 \mathrm{MHz}$, with typical $60 \mathrm{MHz}$ duplex spacing.

It is possible to observe the two radiation maxima corresponding to the two targeted operating bands. The low frequency one is lower than the higher one. This is attributed to the specific coupling between resonating parasitic structures. Loss of radiation efficiency seems to be a consequence of this coupling.

Around $900 \mathrm{MHz}$, the antenna is not yet electrically small and efficiency is higher than $65 \%(\mathrm{ESR}=0 \Omega)$ over a wide band without thin tuning. When frequency is decreased towards a setting around $700 \mathrm{MHz}$, the dual narrow bands are easily observed and efficiency values are lower than at 900 $\mathrm{MHz}$, around $18 \%$ at $700 \mathrm{MHz}$ and $50 \%$ at $760 \mathrm{MHz}(\mathrm{ESR}=$ $0 \Omega$ ). By reaching the $600 \mathrm{MHz}$ bands, the SCA capacitors are 
$0.85 \mathrm{pF}$ which corresponds to the maximal value; the efficiency drops down to $8 \%$ at $600 \mathrm{MHz}$ (respectively $28 \%$ at $630 \mathrm{MHz}$ ). It is interesting to observe the effect of the ESR increase on the antenna radiation efficiency. A quite similar radiation efficiency is observed at different frequencies. The efficiency loss doesn't seem to be as high as for the notch antenna in [3] versus ESR value, typically 10 points loss for ESR of $2 \Omega$.

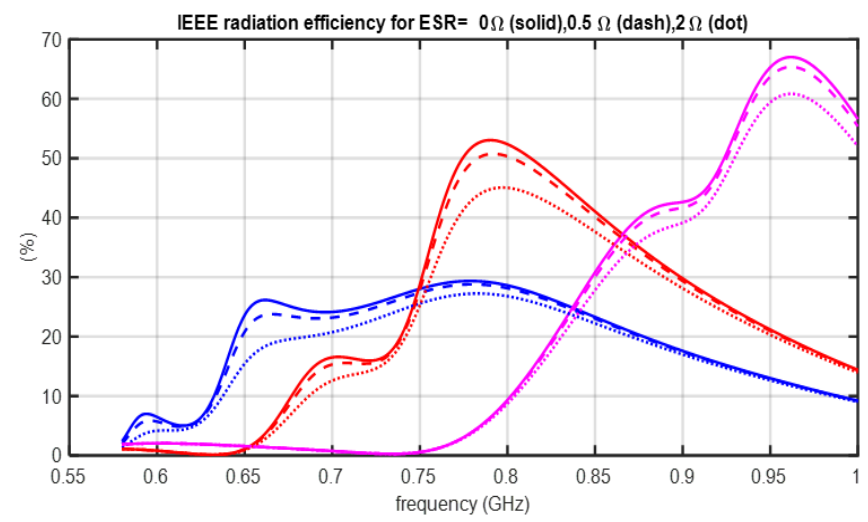

Fig. 3. DTBA simulated IEEE radiation efficiency for three aperture tuning settings for $900-960 \mathrm{MHz}$ (blue), $700-760 \mathrm{MHz}$ (red), and 600-660 MHz (magenta) and for three value of SCA ESR : $0 \Omega$ (solid), $0.5 \Omega$ (dash), $2 \Omega$ (dot)

\section{PROTOTYPE CHARACTERIZATION}

The DTBA prototype is made with a FR4 substrate for the cellular-like board and a flexible thin Kapton substrate for the radiating element. A 3D-printed ABS plastic is used to keep the flexible in the proper folding. The two SCA are mounted on the board whose incorporate the control interface and a SMA connector properly placed at the center and orthogonal to the PCB. Indeed, we have paid attention to the ESA measurement conditions with the development of a test bench dedicated to the electrically small antenna (RF/optical transducer and no metallic wires from the tunable AUT) in CEA LETI anechoic chamber.

Notice that all input impedances are plotted on the antenna feeding plane (that is at the end of the $50 \Omega \mathrm{CPW}$ line).

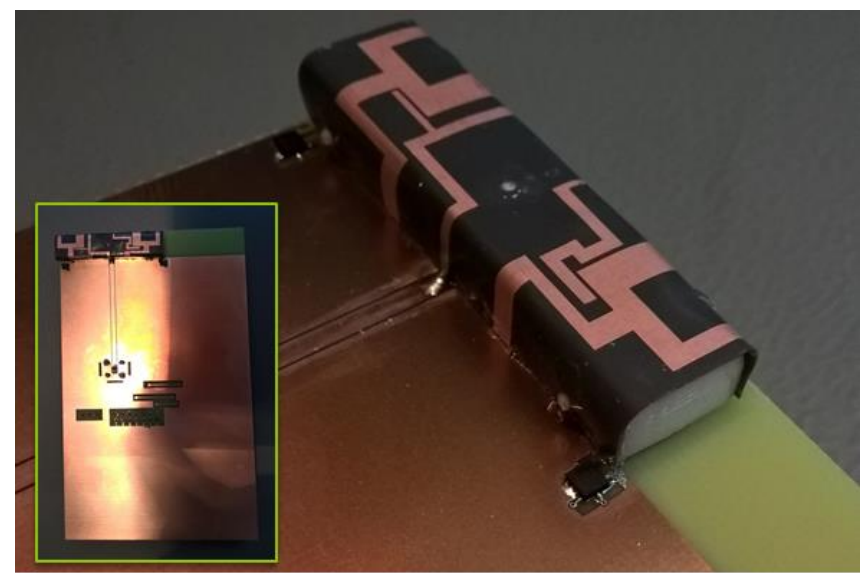

Fig. 4. DTBA prototype with zoom on SCA integration on the PCB board

\section{A. DTBA set in mono resonance mode}

Fig. 5, the measured return loss of DTBA is plotted for various pairs of identical SCA1-SCA2 states (states $i-i$ ) regarding frequencies over [500-1000] MHz. This is called the mono resonance mode The input impedance, real (b) and imaginary (c) parts, allows to observe a single antenna resonance. Notice that the DTBA is supposed to be connected to a tunable matching network, hence here the $50 \Omega$ impedance matching is not yet retrieved, mainly due to a capacitive effect which is frequency-dependent. Nevertheless, the frequency range resonance is between $616 \mathrm{MHz}$ for (states 31-31) and $930 \mathrm{MHz}$ (states 0-0). Notice the thin tunable frequency step by comparing resonance at state 30-30 (solid blue) with those at 31-31 (dashed blue).

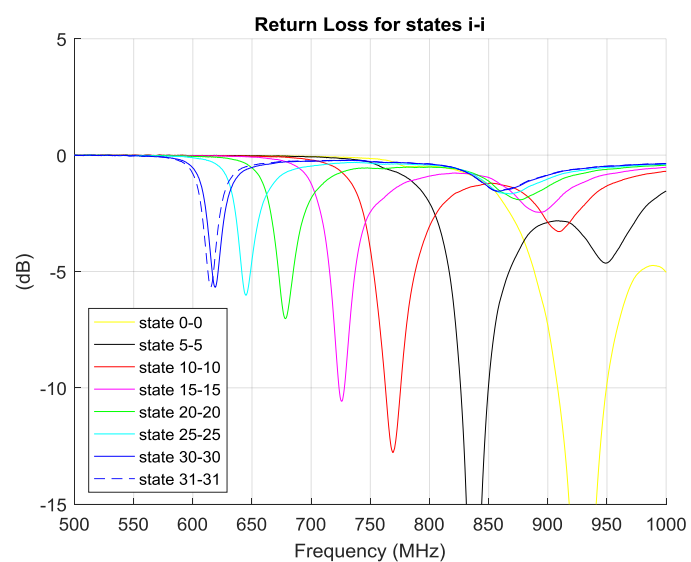

(a)

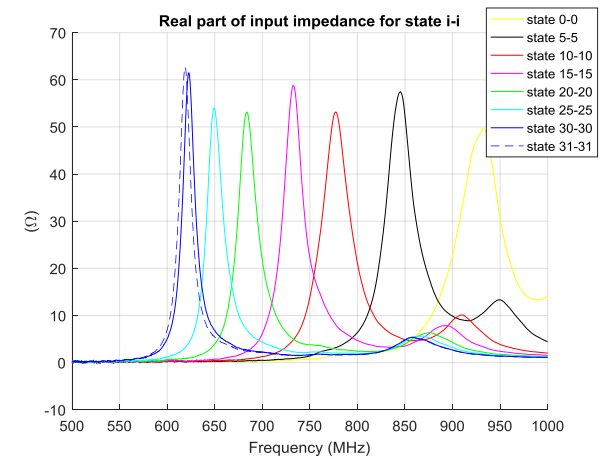

(b)

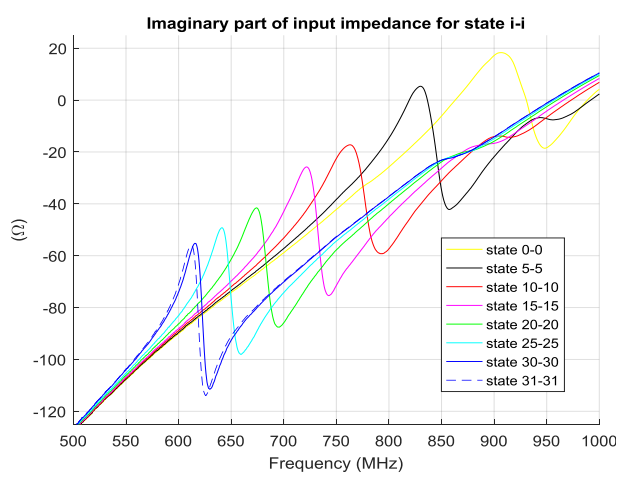

(c)

Fig. 5. Measured DTBA return loss (a) and input impedance real part (b) and imaginary part (c) for different values of states $i-i$ 


\section{B. DTBA set in dual resonance mode}

In this section the dual narrow bands setting is presented. The key advantage of this antenna is to be able to access two narrow independent frequency bands on the $616-940 \mathrm{MHz}$ band. The LTE700 band $\left(\mathrm{n}^{\circ} 28\right)$ is selected to demonstrate this. Fig. 6, the spectrum allocation is depicted for the four French network providers that use this band. Notice a maximum of $10 \mathrm{MHz}$ bandwidth is allocated to one company.

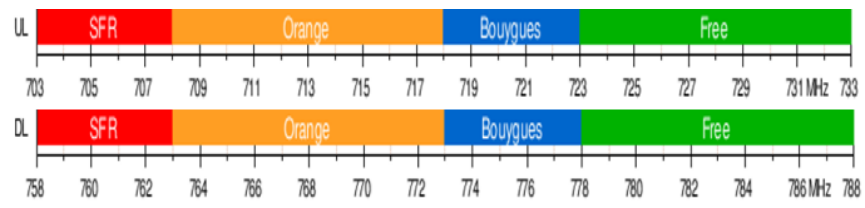

Fig. 6. French ARCEP allocation for the LTE $700 \mathrm{MHz}$ (E-UTRA n ${ }^{\circ} 28$ FDD) band

The DTBA is set to these four bands and the measured return loss and input impedances are plotted in Fig. 7. The $55 \mathrm{MHz}$ duplex spacing is retrieved and the two (UL and DL) resonances are accurately set thanks to the two SCA. The instantaneous bandwidth is delicate to analyze without the TMN, but a $10 \mathrm{MHz}$ bandwidth is currently supported for $3 \mathrm{~dB}$ return loss threshold. The TMN design (not presented in this paper) shows that a mere Pi configuration succeeds to match both resonances with a single set of states. Notice how the DL bands are larger and better matched compared to the UL ones. This is explained by the antenna miniaturization for the lower frequency bands.

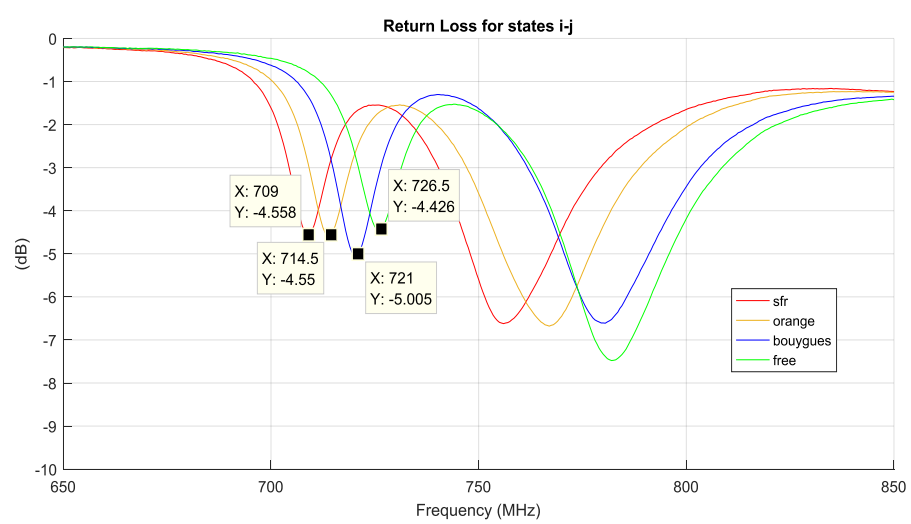

(a)

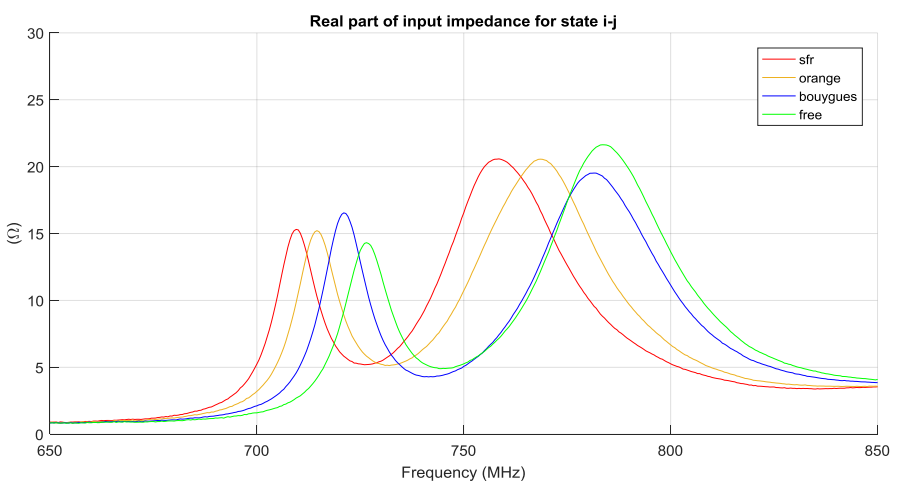

(b)

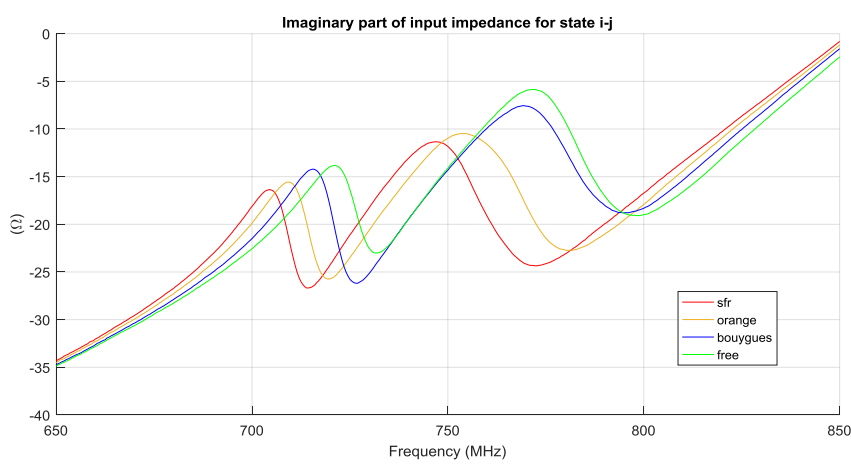

(c)

Fig. 7. Measured DTBA return loss (a), real part (b), and imaginary part (c) for dual band FDD matching in the LTE 700 band for the four French network providers

\section{DTBA radiation pattern in mono resonance mode}

Worst case configuration (lowest frequency: $616 \mathrm{MHz}$ (DSCA states 31-31)) is measured and shown in Fig. 8. The plotted realized gain takes into account the mismatch losses which will be reduced once the matching network will be implemented. At $616 \mathrm{MHz}$, the return loss is about $-6 \mathrm{~dB}$ that is $1.25 \mathrm{~dB}$ mismatch loss. Both co and cross polarizations are observed for three elevation cut-planes. The dipole-like radiation pattern is well retrieved with nulls in the copolarization axis.

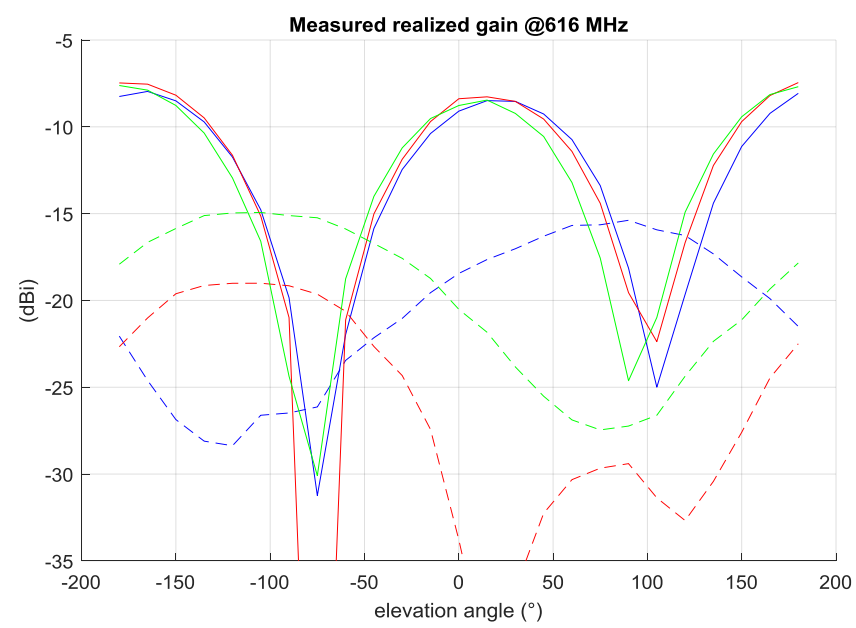

Fig. 8. Measured DTBA realized gain pattern for states 31-31 (616 Mhz) for co-polarization (solid) and cross-polarization (dashed) for three elevation cut-plane: $0^{\circ}$ (blue), $90^{\circ}$ (red), and $45^{\circ}$ (green)

The antenna radiation efficiency characterization is not easy to carry on accurately. Here the integration of realized gain over a sphere is implemented with usual omnidirectionnality validations (Fig. 8). Three cut-planes are used to estimate the total efficiency level around the resonance $(616 \mathrm{MHz})$ which also takes into account the mismatch losses. Fig. 9, the estimated value is $11 \%$ close to the $13 \%$ predicted by the EM simulation on CST MWS with the SCA electrical circuit model. 
Despite a poor efficiency value, and waiting for additional efficiency measurements; two points can be noticed. The first one is the pretty good accuracy of the model: only two SCA states offset that is $1.3 \%$ error on predicted resonance frequency. Moreover, the total efficiency maximum level is also accurately retrieved ( 2 points difference). The confidence on the EM simulation tool and SCA model are key points for future development.

The second point is that the matching network will compensate the $-6 \mathrm{~dB}$ mismatching which corresponds to $1.25 \mathrm{~dB}$ margin on both gain and efficiency. Thus we could expect to reach $15 \%$ maximum level for the efficiency and get a more accurate estimation of the instantaneous bandwidth for this strong miniaturization effort. This will be compared with the $10 \%$ total efficiency of a typical passive antenna.

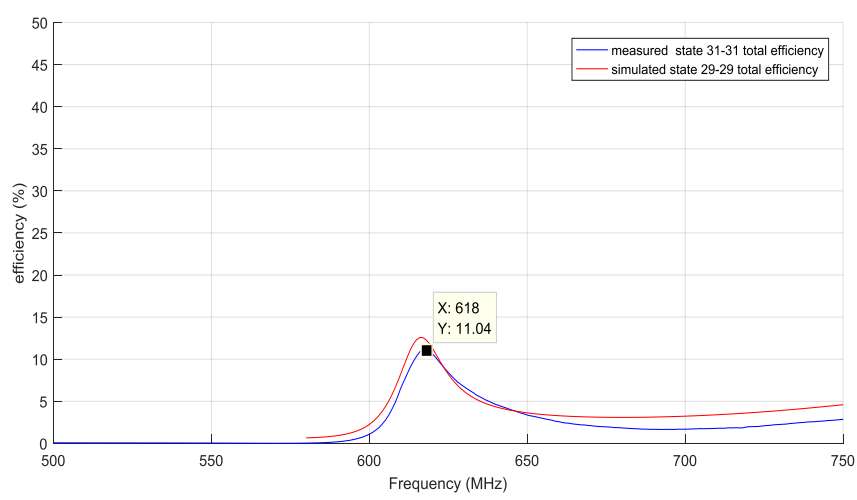

Fig. 9. DTBA simulated and measured total radiation efficiency set at $616 \mathrm{MHz}$

\section{CONCLUSIONS}

To conclude on the DTBA design, the current performance is promising because with small tuning ratio (Cmax/Cmin of 3) and despite a SCA ESR that reaches a $2.5 \Omega$ value, a very miniaturized antenna may be compliant with any LTE FDD standard over a $600-1000 \mathrm{MHz}$ range. It has been demonstrated the quite simple positioning of two resonances on the $600-960 \mathrm{MHz}$ frequency band with variable duplex spacing from $30 \mathrm{MHz}$ to $60 \mathrm{MHz}$. More important, this highly coupled antenna structure seems to be less sensitive to ESR value compared to a typical tunable notch.

We observe a lower radiation efficiency reduction with ESR increase. However, global antenna performance seems to be similar with the passive antenna. The dual band narrow band design has revealed a significant difference in efficiency despite the frequency proximity. Some analysis and optimizations are still needed to get equivalent efficiency on both resonances, which are expected at the highest magnitude.

In order to be competitive regarding passive antenna, two applications are identified: to target the lower than $700 \mathrm{MHz}$ LB for the current smartphone form-factor, or the current 700$960 \mathrm{MHz}$ band but with shrunk form-factor (e.g. cellular watch). On both of them the current passive solution won't be competitive in terms of antenna performance.
The future works will also address the other upper frequency bands with the same antenna structure. Efficiency performance in the dual resonance mode will be shown during the conference.

\section{ACKNOWLEDGMENT}

The authors thank the French BPI to fund a part of this work in the frame of the FELIN project and François Alcouffe for the experimental characterization.

\section{REFERENCES}

[1] 3GPP TR 36.755 V1.0.0 (2017-09), Technical Specification Group RAN US $600 \mathrm{MHz}$ Band for LTE; (Release 15), chap. 5.1,

[2] R. Whatley, T. Ranta and D. Kelly, "RF Front-End Tunability for LTE Handset Applications", 2010 IEEE CSICS, Monterey, CA, 2010

[3] E. Ben Abdallah, D. Nicolas, S. Bories, A. Giry and C. Delaveaud, "A tunable miniaturized notch antenna for low-band LTE applications", 2016 10th EuCAP, Davos, 2016, pp. 1-5.

[4] D. Nicolas et al., "Study of SOI CMOS tunable capacitor architectures and application to antenna aperture tuning," 2016 IEEE MTT-S IMS, San Francisco, CA, 2016, pp. 1-4

[5] Le-Huy Trinh, "Recongurable antennas for mobile phone and WSN applications", PhD thesis , Universite Nice Sophia Antipolis, 2015

[6] M. Stanley, Y. Huang, H. Wang, H. Zhou, Z. Tian and Q. Xu,., "A Novel Reconfigurable Metal Rim Integrated Open Slot Antenna for Octa-Band Smartphone Applications" in IEEE Trans. on Ant. \& Propag., vol. 65, no. 7, pp. 3352-3363, July 2017.

[7] Ben Abdallah, E, et al., "Large-Signal Analysis and Characterization of a RF SOI-Based Tunable Notch Antenna for LTE in TV White Space Frequency Spectrum”, 11th Int. Conf. CROWNCOM 2016 Proceedings, Springer International Publishing

[8] P. Bahramzy, O. Jagielski, S. Svendsen, P. Olesen and G. F. Pedersen, "Aspects of High-Q Tunable Antennas and Their Deployment for 4G Mobile Communications", in IEEE Antennas and Propagation Magazine, vol. 58, no. 4, pp. 70-81, Aug. 2016. 